\title{
Special issue on behavior computing
}

\author{
Longbing Cao • Philip S Yu • Hiroshi Motoda • \\ Graham Williams
}

Published online: 19 June 2013

C) Springer-Verlag London 2013

Behavior is ubiquitous, widely seen from human being, machine and object-related activities and systems. Different from so-called data, behavior forms an intrinsic part of an underlying system and its problems. An abstract behavior [2,10] can be described as a four-tuple object, consisting of actor (subject and/or object), operation (activity and activity properties), relation (interactions) and context (including environment). In deeply understanding behaviors [2], interaction, coupling relationships, semantics, dynamics, change, and impact and utility [5] are important factors to consider.

Behavior Computing (or Behavior Informatics) [3,4] is an emerging field which studies behaviors and behavior-oriented systems from the perspectives of informatics and analytics, aiming to build formal methods and computational theories and techniques for behavior representation, processing and engineering. It aims at inventing and developing computing methodologies, techniques and tools for modeling, representing, reasoning about and checking behavior-oriented systems, for the modeling, analysis, discovery and learning of

L. Cao $(\varangle)$

Advanced Analytics Institute, University of Technology Sydney, Sydney, Australia e-mail: longbing.cao@uts.edu.au

P. S. Yu

Department of Computer Science, University of Illinois at Chicago, Chicago, IL, USA

e-mail: psyu@uic.edu

H. Motoda

Air Force Office of Scientific Research, Asian Office of Aerospace Research and Development, Tokyo, Japan

e-mail: motoda@ar.sanken.osaka-u.ac.jp

H. Motoda

Air Force Research Laboratory, Chicago, IL, USA

G. Williams

Australian Taxation Office, Canberra, Australia

e-mail: graham.williams@togaware.com 
dynamics, networking, group/community formation and deformation, divergence and convergence, pattern and exception, impact, risk and utility, and for the management and emergence of behaviors and behavioral systems.

Behavior computing enables the uncovering of more information and semantics about interior and exterior, individual and collective, implicit and explicit, qualitative and quantitative, and holistic and reductionistic aspects and perspectives about the formation, development, evolution/dynamics, change/revolution, and influence of a system (either individual or networked) and its relevant context and environment. For this, behavior computing needs to involve ubiquitous intelligence embedding and surrounding behaviors and behavioral systems, from behavior operation, data, human, domain (including semantics), organizational, social, cultural and network perspectives.

The above aims and objectives are far beyond the classic efforts made in behavioral science [6,7], social science, and specifically behavioral finance and economics [1]. In fact, there are very few books $[4,9]$ available on behavior analytics from the informatics perspective. This special issue on Behavior Computing aims to raise the need for and opportunities of conducting deep behavior analytics. The collected papers reflect a partial picture of the state-of-the-art research and development in behavior computing and behavior informatics. Through a very rigorous and competitive selection process, six papers are included in this special issue. They involve the following key aspects of behavior computing: group and collective behavior modeling, event extraction, detection and pattern mining, mobile and semantic trajectory analysis, sequential behavior pattern mining and classification, and trust modeling for Web behaviors. These papers address behavioral applications in social network and media, Web services, video objects, mobile devices and services, and customer relationship management.

Behaviors of an individual or a group of people form behavior sequences, which often present various characteristics and challenges in addition to the ordering information. Typically, behavior sequences exhibit features such as time interval between elements in a sequence, couplings between sequences from either one or different actors, simple or complex interactions and relations between sequences or between subsequences. Tsai, Chen and Chien present a paper on incorporating the time-interval feature into constructing behavior sequences. The time-interval sequence of a customer contains not only the temporal order of activities but also the time-interval information between them. They further build a timeinterval sequence classifier, optimized by the particle swam optimization (PSO) algorithm, to classify time-interval behavior sequences. The proposed approach is tested on customer relationship management applications.

In practice, events are widely involved in business, natural, social and artificial systems, in particular, social network systems. An event refers to a specific behavior occurring at a certain place and time. Specifically, event detection, extraction and tracking plays an increasingly important role in understanding and interpreting behaviors and behavioral system dynamics and significant changes. While event extraction and detection has been researched for a while, Nguyen, Phung, Adams and Venkatesh claim that none of this work has considered sentiment information as an underlying cause for event occurrence. Assuming real-world events often involve shared emotional responses from a large population, they leverage the blogosphere as a sentiment 'sensor' to extract events. Tested on millions of blog posts ground truthed with mood labels, their sentiment-based approach formulates a high-order emotional measure, with methods for extracting events based on these time series signals.

Further, Wang, Li, Jiang and Shi introduce their work on detecting sophisticated events such as car parking in video by involving high-level semantic information. Their approach combines low-level visual features and high-level semantic knowledge to detect specific 
semantic trajectory-based events with frequent semantic event (trajectory) patterns by frequent sequence analysis.

Movement is a typical type of mobility behavior. A series of movements form a movement trajectory. Mobile devices and services produce an increasing number of movements. The low-level raw movement trajectories mainly presents geographic coordinates and dynamics. The work investigated by Renso, Baglioni, Macedo, Trasarti and Wachowicz addresses the need for extracting mobility behavioral patterns on low-level movement trajectories and embeds the semantic interpretation of the mined behavioral patterns. Their work is based on the proposed Semantic-Enriched Knowledge Discovery Process, which integrates inductive and deductive reasoning, for handling the syntactic and semantic complexity of movement patterns in order to support meaningful interpretations of human behaviors.

Movement trajectories, forming movement sequences of behavioral actors, can be more informative by attaching scenario and semantic information related to or driving the movements, such as shopping information associated with mobile services. This drives the need to indicate the utility of a movement or movement sequence. The utility of behaviors, namely business concerns, such as profitability of shopping, shows the business interest associated with mobility behavior patterns. This forms high utility mobility sequential behavioral pattern mining. Shie, Hsiao and Tseng explore this particular issue. They introduce three algorithms to discover high utility mobile sequences in mobile commerce data.

Behavior utility discloses the business concerns accompanying a behavior or behavioral sequence. Another interesting question is, how trustworthy are given behaviors, such as Web services behaviors, how trustful they are. Informally, the trust (related to reputation) of a behavior reflects the consistency between the expected and the actual performance after the behavior execution. In the last paper, Yahyaoui and Zhioua investigate the trust patterns of Web services. They address the bootstrapping issue, namely the assignment of initial trust values to unknown Web services, by proposing a hidden Markov model pattern-based classification approach to offer a global view of possible behaviors of Web services.

The above six papers only capture some specific and recent research studies on behavior computing. With the widespread appearance of behaviors and behavior-oriented applications and services, in particular, mobile devices and services, social networks and social media, and the value recognition of studying behaviors from the informatics and analytics perspectives, behavior computing and behavior informatics exhibit powerful and complementary advantages over classic behavior science and domain-specific behavior finance/economics. The integrative exploration of behavior informatics with social informatics [8] complements existing scientific achievements and provides unprecedented and effective theories and tools for an in-depth understanding of complex business, network, social, cultural, economic, governmental and political issues and challenges.

\section{References}

1. Burton E, Shah S (2013) Behavioral finance: understanding the social, cognitive, and economic debates. Wiley, New York

2. Cao LB (2010) In-depth behavior understanding and use: the behavior informatics approach. Inf Sci 180(17):3067-3085

3. Cao L, Yu P (2009) Behavior informatics: an informatics perspective for behavior studies. Intell Inf Bull 10(1):6-11

4. Cao L, Yu P (2012) Behavior computing: modeling, analysis, mining and decision. Springer, Berlin

5. Cao L, Zhao Y, Zhang C (2008) Mining impact-targeted activity patterns in imbalanced data. IEEE Trans Knowl Data Eng 20(8):1053-1066 
6. Fishbein M, Ajzen I (2009) Predicting and changing behavior: the reasoned action approach. Psychology Press, London

7. Fisher W, Piazza C, Roane H (eds) (2011) Handbook of applied behavior analysis. The Guilford Press, New York

8. IEEE Task Force on Behavior and Social Informatics and Computing. www.bsic.info

9. Liu H, Salerno J, Young MJ (eds) (2008) Social computing, behavioral Modeling, and prediction. Springer, Berlin

10. Wang C, Cao L (2012) Modeling and analysis of social activity process. In: Cao L, Yu PS (eds) Behavior computing. Springer, Berlin, pp 21-35

\section{Author Biographies}

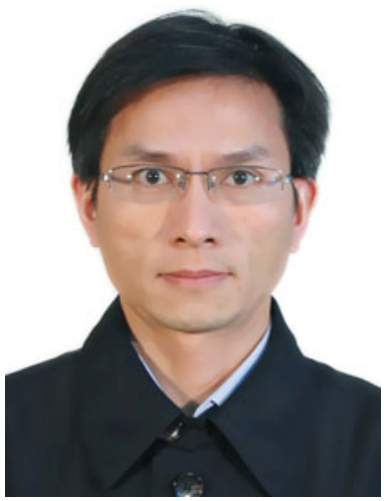

Longbing Cao is a Professor at the University of Technology Sydney, and the Research Leader of the Data Mining Program at the Australian Capital Markets Cooperative Research Centre. He was awarded one Ph.D. degree in Intelligent Sciences and another in Computing Sciences. His research interests include behavior informatics, data mining and machine learning and their applications, multi-agent technology, open complex intelligent systems and agent mining. He has been recognized for his wide engagements in big data analytics and behavior analytics for many different domains and areas including banking, social security, education, telecommunication, insurance, financial markets and government services.

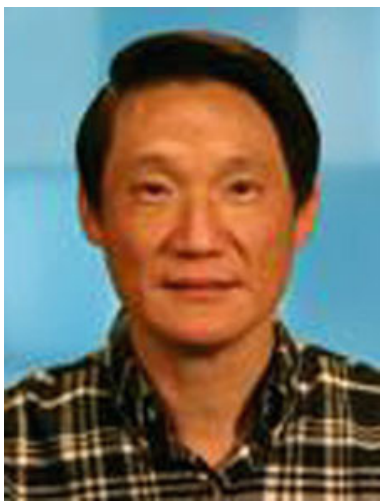

Philip S. Yu is a Professor in Computer Science at the University of Illinois at Chicago and also holds the Wexler Chair in Information Technology. Dr. Yu spent most of his career at IBM, where he was manager of the Software Tools and Techniques group at the Watson Research Center. His research interest is on big data, including data mining, data stream, database and privacy. He has published more than 740 papers in refereed journals and conferences. He holds or has applied for more than 300 US patents. Dr. Yu is a Fellow of the ACM and the IEEE. He is the Editor-in-Chief of ACM Transactions on Knowledge Discovery from Data. He was the Editor-in-Chief of IEEE Transactions on Knowledge and Data Engineering (2001-2004). He received an IEEE Technical Achievement Award "for pioneering and fundamentally innovative contributions to the scalable indexing, querying, searching, mining and anonymization of big data" (2013). He received a Research Contributions Award from IEEE Intl. Conference on Data Mining (2003). He was an IBM Master Inventor. Dr. Yu received his Ph.D. degree in E.E. from Stanford University. 


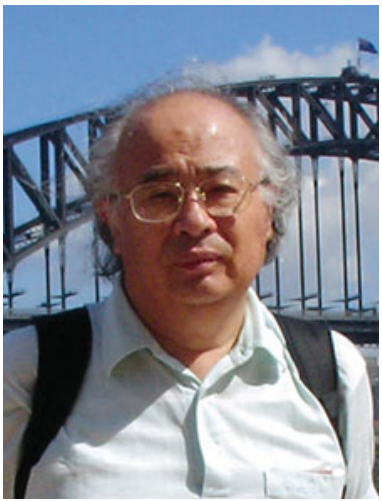

Hiroshi Motoda is a Professor Emeritus of Osaka University and a scientific advisor of Asian Office of Aerospace Research and Development, Air Force Office of Scientific Research, US Air Force Research Laboratory. His research interests include machine learning, data mining, social network analysis and artificial intelligence. $\mathrm{He}$ is a cofounder of PAKDD and ACML, and is a fellow of JSAI.

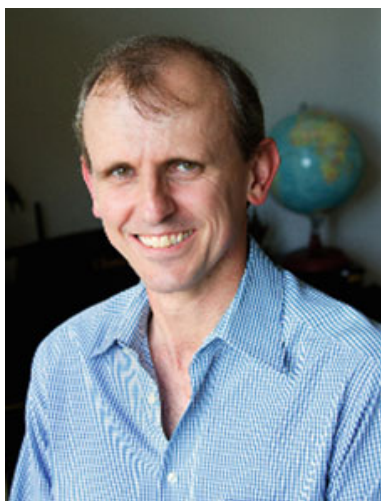

Graham Williams is a director of data miner with the Australian Taxation Office, an adjunct professor with the Australian National University and the University of Canberra, and an International Visiting Professor with the Chinese Academy of Sciences. His research interests include ensemble learning, data mining and artificial intelligence. He is chair of the PAKDD and the AusDM steering committees. 